\title{
Eficácia do Herbicida Oxyfluorfen com a Cobertura de Palha no Controle de Plantas Daninhas ${ }^{1}$
}

\author{
Efficacy of Oxyfluorfen with Straw Cover in controlling Weeds
}

\begin{abstract}
NEGRISOLI, E. ${ }^{2}$, CORREA, M.R. ${ }^{3}$, ROSSI, C.V.S. ${ }^{4}$, CARBONARI, C.A. ${ }^{5}$, VELINI, E.D. ${ }^{6}$ e PERIM, L. ${ }^{7}$
RESUMO - A palha pode alterar a dinâmica do herbicida oxyfluorfen no sistema de canacrua; assim, este trabalho teve por objetivo avaliar a eficácia do oxyfluorfen em condições de casa de vegetação, com a cobertura de palha, no controle das plantas daninhas que ocorrem em cana-crua. Foram avaliadas as espécies de Brachiaria decumbens, Ipomoea grandifolia, Ipomoea quamoclit e Merremia cissoides. A dose utilizada do oxyfluorfen foi de $3 \mathrm{~L} \mathrm{ha}^{-1}$ (720 g i.a. ha $\left.{ }^{-1}\right)$. Os tratamentos constaram de diferentes posicionamentos do herbicida e condições antes e após a aplicação. A porcentagem de controle das plantas daninhas foi avaliada aos 12, 20, 26, 33 e 38 DAA. Os maiores indices de controle aos 38 DAA, independentemente da planta daninha estudada, foram alcançados quando se aplicou oxyfluorfen em tratamentos com a presença de palha em cobertura, com ou sem umidade. No entanto, os resultados obtidos nos demais tratamentos mostraram-se eficientes no controle das diferentes espécies de plantas daninhas estudadas.
\end{abstract}

Palavras-chave: cana-de-açúcar, palha, planta daninha.

\begin{abstract}
Straw can change the dynamics of the herbicide oxyfluorfen in the raw sugarcane system. Thus, this study aimed to assess the effectiveness of oxyfluorfen with straw cover in controlling weeds in raw sugarcane under greenhouse conditions. The species Brachiaria decumbens, Ipomoea grandifolia, Ipomoea quamoclit and Merremia cissoids were evaluated and the dose used was oxyfluorfen $3 \mathrm{~L} \mathrm{ha}^{-1}$ (720 g i.a. ha $\mathrm{h}^{-1}$ ). Treatments consisted of different herbicide positions and conditions before and after application. Weed control percentage was evaluated at 12, 20, 26, 33 and 38 DAA. The highest control rates at 38 DAA, regardless of the weed studied, were reached when oxyfluorfen was applied in treatments with the cover straw, with or without humidity. However, the results obtained in the other treatments were effective in controlling the different weed species studied.
\end{abstract}

Keywords: sugarcane, straw, weed.

\section{INTRODUÇÃO}

A presença da palha é apenas uma das barreiras para o uso de herbicidas com ação exclusiva ou preferencial no solo. Embora o solo esteja coberto com uma camada de palha, sabe-se que determinadas espécies de plantas daninhas, ao germinarem, irão vencer essa barreira física e se estabelecer no canavial, o que pode ser resultado do fato de alguns herbicidas serem mais retidos pela cobertura morta (Rossi, 2004) e, consequentemente, terem sua eficácia reduzida.

1 Recebido para publicação em 2.6.2008 e na forma revisada em 18.2.2009.

2 Engo-Agro- Dr., TechField-Nupam/FCA-Unesp, Caixa Postal 237, 18603-970 Botucatu-SP, <eduardo.negrisoli@gmail.com>; ${ }^{3}$ Eng-o-Agr ${ }^{\circ}$, Dr., TechField-Nupam/FCA-Unesp, Botucatu-SP, <marcelorcorrea@uol.com.br $>$; ${ }^{4}$ Engo-Agr ${ }^{\circ}$, Dr., TechField-Nupam/ FCA-Unesp, Botucatu-SP, Caixa Postal 237, 18603-970, Botucatu-SP, <cavsragro@msn.com>; 5 Engo-Agr ${ }^{\circ}$, M.Sc., FCA-Unesp, Botucatu-SP, <carbonari@fca.unesp.br>; ${ }^{6}$ Prof. Dr., Dep. de Agricultura FCA/Unesp - Botucatu-SP, Fazenda Experimental Lageado, Caixa Postal 237, 18603-970, Botucatu-SP, <velini@fca.unesp.br>; 7 Graduação do curso de Agronomia da FCA-Unesp, Botucatu-SP, <lperim@fca.unesp.br> 
O acréscimo do teor superficial de matéria orgânica no solo, menos evidente em canacrua do que em plantio direto, em função da movimentação mínima no momento da colheita mecanizada, associada ao preparo e sulcamento quando da reimplantação da cultura, propicia a adsorção dos herbicidas, limitando a sua eficiência (Tofoli, 2004).

Quando aplicado sobre a palhada, o herbicida é interceptado pela superfície da palha ali depositada e torna-se vulnerável à degradação causada pela volatilização e/ou fotodecomposição, até ser lixiviado para o solo (Locke \& Bryson, 1997). Lamoreaux et al. (1993) ressaltaram que a lavagem de herbicidas da palha para o solo é dependente da capacidade desses resíduos em cobrir o solo e reter os herbicidas; da solubilidade do produto aplicado; e do período em que a área permanece sem chuva após a aplicação do produto. A partir do momento que esses herbicidas atingem o solo, apresentam maior distribuição e persistência, devido aos canais formados pelos restos vegetais (formando vias preferenciais de escoamento) ou por organismos do solo (minhocas) e, principalmente, pela amenização dos processos de degradação (Jones Jr. et al., 1990; Sorenson et al., 1991).

Correia \& Durigan (2004) estudaram os efeitos da cobertura do solo, com $0,5,10$ e $15 \mathrm{t}$ ha ${ }^{1}$, sobre a emergência de seis espécies de plantas daninhas (Brachiaria decumbens, Digitaria horizontalis, Sida spinosa, Ipomoea grandifolia, Ipomoea hederifolia e Ipomoea quamoclit) e constataram que a cobertura do solo com 5,10 e $15 \mathrm{t} \mathrm{ha}^{-1}$ inibiu a emergência de plântulas de $B$. decumbens e $S$. spinosa, sendo o mesmo observado para $D$. horizontalis submetida a 10 e $15 \mathrm{t} \mathrm{ha}^{-1}$. No entanto, para I. grandifolia e I. hederifolia, o número de plantas emersas não diferiu entre as quantidades de palha. Por sua vez, a presença da cobertura morta com palha de cana incrementou a emergência de plântulas de I. quamoclit.

A palha afeta drasticamente o estabelecimento de plantas daninhas em áreas cultivadas de diferentes maneiras, entre as quais podem-se citar a limitação de variação da temperatura na superficie do solo; a formação de uma barreira física a ser transposta pela planta em germinação; o aumento da quantidade de microrganismos que podem decompor as sementes destas plantas; e os possiveis efeitos alelopáticos que inibem a germinação. Apesar disso, algumas plantas daninhas, como Ipomoea grandifolia e Euphorbia heterophylla, não têm sua germinação inibida pelas quantidades de palha de cana-de-açúcar normalmente encontradas em campo (Martins et al., 1999; Velini \& Negrisoli, 2000; Correia \& Durigan, 2004; Negrisoli, 2005).

As convolvuláceas, principalmente as pertencentes aos gêneros Ipomoea e Merremia, destacam-se entre as plantas daninhas que podem causar sérios danos à cultura da canade-açúcar, especialmente em áreas de canacrua. Além de competirem com a planta cultivada, podem interferir nas práticas culturais, sobretudo na colheita mecanizada, reduzindo sua eficiência (Azania et al., 2002).

Este trabalho teve como objetivo avaliar a eficácia do herbicida oxyfluorfen, em casa de vegetação, quando aplicado em diferentes posicionamentos em relação à camada de palha de cana-de-açúcar e à simulação ou não de chuva após a aplicação.

\section{MATERIAL E MÉTODOS}

O experimento foi realizado em casa de vegetação no Núcleo de Pesquisas Avançadas em Matologia (NUPAM), pertencente ao Departamento de Produção Vegetal da Faculdade de Ciências Agronômicas/UNESP Botucatu-SP.

O solo utilizado, classificado como de textura média, foi inicialmente seco à sombra por um período de 48 horas. Após esse período, foi peneirado em peneira com malha de 200 mesh, submetido às análises químicas e granulométricas (Tabelas 1 e 2) e adubado de acordo com o resultado da análise, para propiciar melhor germinação das plantas daninhas. Em seguida, o solo foi acondicionado em vasos de 2 L de capacidade, procedendo-se à semeadura com as espécies Brachiaria decumbens, Ipomoea grandifolia, Ipomoea quamoclit e Merremia cissoides. A umidade do solo, medida gravimetricamente, foi mantida durante todo o ensaio em torno de $65 \%$ da capacidade de campo. 
Tabela 1 - Análise química da amostra de solo utilizado nos vasos. Botucatu-SP, 2007

\begin{tabular}{|c|c|c|c|c|c|c|c|c|c|c|}
\hline \multirow{2}{*}{ Solo } & $\mathrm{PH}$ & M.O. & P res. & $\mathrm{K}^{+}$ & $\mathrm{Ca}^{+2}$ & $\mathrm{Mg}^{+2}$ & $\mathrm{H}^{+}+\mathrm{Al}^{+3}$ & $\overline{S B}$ & $\mathrm{~T}$ & \multirow{2}{*}{$\begin{array}{c}\mathrm{V} \\
(\%)\end{array}$} \\
\hline & $\left(\mathrm{CaCl}_{2}\right)$ & $\left(\mathrm{g} \mathrm{dm}^{-3}\right)$ & $\left(\mathrm{Mg} \mathrm{dm}^{-3}\right)$ & \multicolumn{6}{|c|}{$\left(\right.$ mmolc dm $\left.^{-3}\right)$} & \\
\hline $\mathrm{LVd}$ & 4,3 & 19 & 1 & 0,6 & 10 & 4 & 58 & 14,6 & 73 & 21 \\
\hline
\end{tabular}

Departamento de Solos - FCA/UNESP - Botucatu.

Tabela 2 - Análise granulométrica do solo utilizado no experimento. Botucatu-SP, 2007

\begin{tabular}{|c|c|c|c|c|c|c|c|}
\hline \multirow{2}{*}{ Granulometria (\%) } & \multicolumn{6}{|c|}{ Classe de solo } & \multirow{2}{*}{ Classe textural } \\
\cline { 2 - 7 } & \multirow{2}{*}{ Argila } & \multirow{2}{*}{ Limo } & \multicolumn{5}{|c|}{ Areia } \\
\cline { 4 - 7 }$n$ & & & Fina & Média & Grossa & Total & \multirow{2}{*}{ Média } \\
\hline \multirow{2}{*}{ LVd } & 20 & 4 & 22,9 & 35,7 & 17,4 & 76 & 76 \\
\hline
\end{tabular}

Departamento de Solos - FCA/UNESP - Botucatu.

A palha de cana-de-açúcar utilizada neste estudo foi da variedade SP 80-1816, na quantidade de $5 \mathrm{t} \mathrm{ha}^{-1}$, sendo selecionada em razão das informações disponiveis na literatura e de estudos anteriormente realizados, considerando ser essa quantidade uma das condições mais críticas em termos de manejo de plantas daninhas em cana-crua, uma vez que é insuficiente para controlar adequadamente as plantas daninhas, mas o bastante para reter quase que completamente a quantidade de calda aplicada (Velini \& Negrisoli, 2000; Veiga Filho, 2002; Medeiros \& Christoffoleti, 2002).

A pulverização do herbicida e a simulação de chuva foram realizadas por meio de equipamento instalado no laboratório do NuPAM, o qual se constitui de uma estrutura metálica com $3 \mathrm{~m}$ de altura por $2 \mathrm{~m}$ de largura, que permite acoplamento de um "carrinho" suspenso a 2,5 m de altura. A esse carrinho encontramse acopladas duas barras de pulverização: uma responsável pelo sistema de simulação de chuva e a outra pelo sistema de pulverização de defensivos agrícolas, as quais se deslocam por uma área útil de $6 \mathrm{~m}^{2}$ no sentido do comprimento do equipamento. O tracionamento de ambas as barras é realizado por meio de correntes e engrenagens, com o auxílio de um motor elétrico, cujo ajuste é feito por um modulador de frequência, permitindo a obtenção de velocidade constante previamente determinada.
A simulação da chuva foi feita utilizandose uma bomba hidráulica de pressão constante e acionamento automático, a qual bombeia água armazenada de um reservatório, com capacidade para $1.000 \mathrm{~L}$, até a barra e pontas de pulverização responsáveis pela formação de gotas de chuva. A barra de simulação de chuva situada a 1,45 m de altura em relação à superficie das unidades experimentais é constituída por três bicos de pulverização TK-SS-20 de alta vazão, espaçados de $0,5 \mathrm{~m}$ e posicionados de forma a propiciar maior uniformidade de precipitação na área aplicada.

Esse sistema foi operado com velocidade de deslocamento de $0,187 \mathrm{~km} \mathrm{~h}^{-1}$, o que correspondeu a 2,5 Hertz no modulador de frequência, e pressão de trabalho de $0,81 \mathrm{kgf} \mathrm{s}^{-2}$. Essas especificações proporcionaram a produção de gotas artificiais de chuva com diâmetro mediano volumétrico (DMV) de 1.140 micras, conforme informações do fabricante da ponta de pulverização (Spraying Systems Co.), e cada lâmina aplicada correspondeu a aproximadamente 2,5 $\mathrm{mm}$ de chuva.

A barra de pulverização constituiu-se de quatro pontas de pulverização XR110.02 VS, espaçadas de $0,5 \mathrm{~m}$ e posicionadas a $0,5 \mathrm{~m}$ de altura em relação à superficie das unidades experimentais.

Para as pulverizações do herbicida, o sistema foi operado com velocidade de deslocamento de 3,6 $\mathrm{km} \mathrm{h}^{-1}$, o que correspondeu a 45,0 Hertz 
no modulador de frequência, com consumo de calda correspondente a $200 \mathrm{~L} \mathrm{ha}^{-1}$. O equipamento foi operado sob pressão constante de 1,5 bar, pressurizado por ar comprimido. A dose de oxyfluorfen (Goal BR) aplicada foi de 3 L p.c. ha ${ }^{-1}$ (720 gi.a. ha-1).

O controle das espécies de plantas daninhas foi determinado por meio de uma escala visual e percentual de notas, variando de zero a 100. Nesse caso, zero consistia em nenhum controle e 100 era a porcentagem de controle total das espécies daninhas, As avaliações foram realizadas até o desaparecimento ou a estabilização dos sintomas de fitointoxicação (SBCPD, 1995), aos 12, 20, 26, 33 e 38 dias após a aplicação (DAA).

Os dados foram comparados estatisticamente pelo teste de Tukey a $5 \%$ de probabilidade, incluindo-se testemunhas com e sem a palha da cana-de-açúcar, para avaliação do controle proporcionado pelo oxyfluorfen.

Na Tabela 3 são apresentados os tratamentos utilizados no experimento conduzido em vasos com diferentes posicionamentos do herbicida oxyfluorfen.

\section{RESULTADOS E DISCUSSÃO}

Para a espécie Brachiaria decumbens (Tabela 4), independentemente do tratamento testado, observou-se excelente controle desde o início das avaliações, ocorrido aos 12 DAA do herbicida. Inicialmente, observa-se que o tratamento em que o produto foi aplicado sobre a palha e não ocorreu precipitação posterior (tratamento 2), apesar de mostrar média de controle inferior, não apresentou diferença significativa em relação aos demais tratamentos avaliados. A partir disso, com exceção do tratamento em que a aplicação ocorreu sobre a superficie do solo sem a cobertura posterior de palha (tratamento 6), que apresentou médias de $99,5 \%$ aos 20 e 26 DAA, respectivamente, todos os demais mostraram controle total dessa espécie durante a condução do experimento. Em trabalho semelhante, com estudos realizados em casa de vegetação com palha de cana-de-açúcar, Negrisoli et al. (2007a) obtiveram excelente controle de Brachiaria decumbens com aplicação do herbicida amicarbazone.

Considerando as avaliações para a espécie Ipomoea grandifolia (Tabela 5), podem ser observadas excelentes médias de controle desde o início das avaliações. Esses resultados não diferiram entre os tratamentos aos $12 \mathrm{e}$ 20 DAA, com médias variando de $95,3 \%$ a 99,5\%. Negrisoli et al. (2007b) observaram resultados semelhantes, obtendo controle excelente de $I$. grandifolia com aplicação do herbicida tebuthiuron, com o qual todos os tratamentos apresentaram excelente niveis de controle já a partir dos 28 DAA.

A partir de 26 DAA, os tratamentos apresentaram algumas particularidades. O tratamento em que se simulava uma aplicação conjunta com a colheita (tratamento 3 : semeadura + chuva de $30 \mathrm{~mm}+$ aplicação + palha $5 \mathrm{t} \mathrm{ha}^{-1}$ ) mostrou-se estatisticamente menos eficaz em relação aos demais, apesar de apresentar $98 \%$ de controle. Essa tendência foi observada até o final das avaliações. No entanto, esse valor pode ser considerado uma excelente média de controle de espécies de plantas daninhas. Esses resultados foram também observados por Negrisoli et al. (2004) com a aplicação de sulfentrazone sobre a palha

Tabela 3 - Descrição dos tratamentos utilizados no experimento. Botucatu-SP, 2007

\begin{tabular}{|c|c|}
\hline Tratamento & Ordem sequencial de condução \\
\hline 1 & Semeadura + palha $5 \mathrm{t} \mathrm{ha}^{-1}+$ aplicação + chuva de $30 \mathrm{~mm}$ \\
\hline 2 & Semeadura + chuva de $30 \mathrm{~mm}+$ palha $5 \mathrm{t} \mathrm{ha}^{-1}+$ aplicação \\
\hline 3 & Semeadura + chuva de $30 \mathrm{~mm}+$ aplicação + palha $5 \mathrm{t} \mathrm{ha}^{-1}$ \\
\hline 4 & Semeadura + palha $5 \mathrm{t} \mathrm{ha}^{-1}+$ chuva $(30 \mathrm{~mm})+$ aplicação (12 h após) \\
\hline 5 & Semeadura + palha $5 \mathrm{t} \mathrm{ha}^{-1}+$ aplicação + chuva de $2,5 \mathrm{~mm}$ \\
\hline 6 & Semeadura + chuva de $30 \mathrm{~mm}+$ palha $5 \mathrm{t} \mathrm{ha}^{-1}+$ aplicação \\
\hline 7 & Semeadura + chuva de $30 \mathrm{~mm}+$ aplicação \\
\hline
\end{tabular}


de cana, os quais obtiveram excelente controle (100\% aos 28 dias após a aplicação) da espécie I. grandifolia quando ocorreu uma chuva de 20 mm, 24 horas após a aplicação.

Tabela 4 - Controle de Brachiaria decumbens pelo oxyfluorfen na dose de 3,0 L p.c. ha ${ }^{1}$, aplicado em diferentes posicionamentos em relação à palha de cana-de-açúcar. BotucatuSP, 2007

\begin{tabular}{|l|c|c|c|c|c|}
\hline \multirow{2}{*}{ Tratamento } & \multicolumn{5}{|c|}{ Dias após a aplicação (DAA) } \\
\cline { 2 - 6 } & 12 & 20 & 26 & 33 & 38 \\
\hline 1. PAC & $99,8 \mathrm{a}$ & $100,0 \mathrm{a}$ & $100,0 \mathrm{a}$ & $100,0 \mathrm{a}$ & $100,0 \mathrm{a}$ \\
\hline 2. CPA & $99,3 \mathrm{a}$ & $100,0 \mathrm{a}$ & $100,0 \mathrm{a}$ & $100,0 \mathrm{a}$ & $100,0 \mathrm{a}$ \\
\hline 3. CAP & $100,0 \mathrm{a}$ & $100,0 \mathrm{a}$ & $100,0 \mathrm{a}$ & $100,0 \mathrm{a}$ & $100,0 \mathrm{a}$ \\
\hline 4. $\mathrm{PC}_{12 \mathrm{~h}} \mathrm{~A}$ & $100,0 \mathrm{a}$ & $100,0 \mathrm{a}$ & $100,0 \mathrm{a}$ & $100,0 \mathrm{a}$ & $100,0 \mathrm{a}$ \\
\hline 5. PAC 2,5 & $99,5 \mathrm{a}$ & $100,0 \mathrm{a}$ & $100,0 \mathrm{a}$ & $100,0 \mathrm{a}$ & $100,0 \mathrm{a}$ \\
\hline 6. AC & $100,0 \mathrm{a}$ & $99,5 \mathrm{a}$ & $99,5 \mathrm{a}$ & $100,0 \mathrm{a}$ & $100,0 \mathrm{a}$ \\
\hline 7. Test. palha & - & - & - & - & - \\
\hline 8. Test. s/palha & - & - & - & - & - \\
\hline F tratamento & $2,88^{* *}$ & $3,00 * *$ & $3,00 * *$ & - & - \\
\hline CV (\%) & 0,37 & 0,24 & 0,24 & $----\cdot$ & - \\
\hline DMS & 0,84 & 0,53 & 0,53 & - & - \\
\hline
\end{tabular}

Médias seguidas de mesma letra na coluna não diferem estatisticamente entre si pelo teste de Tukey $(\mathrm{P}<0,05)$.

$*$ significativo a $5 \%$ de probabilidade, ${ }^{*}$ significativo a $1 \%$ de probabilidade, ns - não-significativo.

$\mathrm{P}=$ cobertura com $5 \mathrm{tha}^{-1}$ de palha de cana-de-açúcar; $\mathrm{C}=$ chuva de $30 \mathrm{~mm} ; \mathrm{C}_{12 \mathrm{~h}}=$ chuva de $30 \mathrm{~mm} 12$ horas antes da aplicação; $\mathrm{C}_{2,5}=$ chuva de $2,5 \mathrm{~mm} ; \mathrm{A}=$ aplicação de oxyfluorfen.

Tabela 5 - Controle de Ipomoea grandifolia pelo oxyfluorfen na dose de 3,0 L p.c. ha ${ }^{-1}$, aplicado em diferentes posicionamentos em relação à palha de cana-de-açúcar. BotucatuSP, 2007

\begin{tabular}{|l|c|c|c|c|c|}
\hline \multirow{2}{*}{ Tratamento } & \multicolumn{5}{|c|}{ Dias após a aplicação (DAA) } \\
\cline { 2 - 6 } & 12 & 20 & 26 & 33 & 38 \\
\hline 1. PAC & $95,3 \mathrm{a}$ & $98,0 \mathrm{a}$ & $99,8 \mathrm{a}$ & $100,0 \mathrm{a}$ & $100,0 \mathrm{a}$ \\
\hline 2. CPA & $97,8 \mathrm{a}$ & $98,8 \mathrm{a}$ & $99,8 \mathrm{a}$ & $100,0 \mathrm{a}$ & $100,0 \mathrm{a}$ \\
\hline 3. CAP & $95,8 \mathrm{a}$ & $97,5 \mathrm{a}$ & $98,0 \mathrm{~b}$ & $98,0 \mathrm{~b}$ & $98,8 \mathrm{~b}$ \\
\hline 4. PC $_{12 \mathrm{~h}} \mathrm{~A}$ & $97,8 \mathrm{a}$ & $98,8 \mathrm{a}$ & $99,5 \mathrm{a}$ & $100,0 \mathrm{a}$ & $100,0 \mathrm{a}$ \\
\hline 5. PAC 2,5 & $99,3 \mathrm{a}$ & $97,5 \mathrm{a}$ & $98,8 \mathrm{ab}$ & $99,8 \mathrm{a}$ & $99,8 \mathrm{a}$ \\
\hline 6. AC & $96,3 \mathrm{a}$ & $99,5 \mathrm{a}$ & $99,5 \mathrm{a}$ & $100,0 \mathrm{a}$ & $100,0 \mathrm{a}$ \\
\hline 7. Test. palha & - & - & - & - & - \\
\hline 8. Test. s/palha & - & - & - & - & - \\
\hline F tratamento & $2,80^{*}$ & $2,25 *$ & $8,22 * *$ & $61,80^{* *}$ & $12,00 * *$ \\
\hline CV (\%) & 1,86 & 1,09 & 0,49 & 0,20 & 0,29 \\
\hline DMS & 4,05 & 2,39 & 1,09 & 0,46 & 0,65 \\
\hline
\end{tabular}

Médias seguidas de mesma letra na coluna não diferem estatisticamente entre si pelo teste de Tukey $(\mathrm{P}<0,05)$.

* significativo a $5 \%$ de probabilidade, $* *$ significativo a $1 \%$ de probabilidade, ns - não-significativo.

$\mathrm{P}=$ cobertura com $5 \mathrm{t} \mathrm{ha}^{-1}$ de palha de cana-de-açúcar; $\mathrm{C}=$ chuva de $30 \mathrm{~mm} ; \mathrm{C}_{12 \mathrm{~h}}=$ chuva de $30 \mathrm{~mm} 12$ horas antes da aplicação; $\mathrm{C}_{2,5}=$ chuva de $2,5 \mathrm{~mm} ; \mathrm{A}=$ aplicação de oxyfluorfen.
Quanto à espécie Ipomoea quamoclit (Tabela 6), os resultados de controle obtidos pelo oxyfluorfen foram considerados excelentes para essa espécie. Não houve diferença entre os tratamentos em nenhuma das avaliações realizadas. A partir de 20 DAA, os tratamentos T1 (aplicação sobre a palha seguida de chuva de $30 \mathrm{~mm}$ ), T4 (aplicação do herbicida sobre a palha umedecida com chuva ocorrida 12 horas antes) na presença de palha e T6 (aplicação sobre a superficie do solo) com ausência de palha apresentaram controle total dessa espécie. Nos demais tratamentos, os valores médios variaram de 96,5 a 99,8\%. Em condições semelhantes, Corrêa (2006) e Rossi (2007) obtiveram excelentes resultados de controle dessa espécie com aplicação de hexazinona+ diuron (Velpar K GRDA) e metribuzin (Sencor 480), respectivamente.

Como observado para a espécie I. quamoclit, os resultados de controle pelo herbicida oxyfluorfen sobre $M$. cissoides (Tabela 7) foram considerados excelentes, com exceção do tratamento 5, no qual após a aplicação do produto ocorreu precipitação de $2,5 \mathrm{~mm}$, que apresentou menores médias, sendo estatisticamente inferiores para as avaliações ocorridas aos 12 e 20 DAA. Nos demais tratamentos o

Tabela 6 - Controle de Ipomoea quamoclit pelo oxyfluorfen na dose de 3,0 L p.c. ha-1 ${ }^{-1}$ aplicado em diferentes posicionamentos em relação à palha de cana-de-açúcar. BotucatuSP, 2007

\begin{tabular}{|l|c|c|c|c|c|}
\hline \multirow{2}{*}{ Tratamento } & \multicolumn{5}{|c|}{ Dias após a aplicação (DAA) } \\
\cline { 2 - 6 } & 12 & 20 & 26 & 33 & 38 \\
\hline 1. PAC & $98,5 \mathrm{a}$ & $100,0 \mathrm{a}$ & $100,0 \mathrm{a}$ & $100,0 \mathrm{a}$ & $100,0 \mathrm{a}$ \\
\hline 2. CPA & $98,5 \mathrm{a}$ & $99,5 \mathrm{a}$ & $99,5 \mathrm{a}$ & $99,5 \mathrm{a}$ & $99,8 \mathrm{a}$ \\
\hline 3. CAP & $99,0 \mathrm{a}$ & $99,5 \mathrm{a}$ & $99,5 \mathrm{a}$ & $99,5 \mathrm{a}$ & $99,8^{\mathrm{a}}$ \\
\hline 4. $\mathrm{PC}_{12 \mathrm{~h}} \mathrm{~A}$ & $99,5 \mathrm{a}$ & $100,0 \mathrm{a}$ & $100,0 \mathrm{a}$ & $100,0 \mathrm{a}$ & $100,0 \mathrm{a}$ \\
\hline 5. PAC 2,5 & $96,5 \mathrm{a}$ & $98,0 \mathrm{a}$ & $99,0 \mathrm{a}$ & $99,5 \mathrm{a}$ & $99,8 \mathrm{a}$ \\
\hline 6. AC & $99,3 \mathrm{a}$ & $100,0 \mathrm{a}$ & $100,0 \mathrm{a}$ & $100,0 \mathrm{a}$ & $100,0 \mathrm{a}$ \\
\hline 7. Test. palha & - & - & - & - & - \\
\hline 8. Test. s/palha & - & - & - & - & - \\
\hline F tratamento & $1,86^{\mathrm{ns}}$ & $2,70^{*}$ & $2,00^{\mathrm{ns}}$ & $1,80^{\mathrm{ns}}$ & $0,60^{\mathrm{ns}}$ \\
\hline CV $(\%)$ & 1,60 & 0,95 & 0,58 & 0,41 & 0,35 \\
DMS & 3,54 & 2,12 & 1,30 & 0,92 & 0,79 \\
\hline
\end{tabular}

Médias seguidas de mesma letra na coluna não diferem estatisticamente entre si pelo teste de Tukey $(\mathrm{P}<0,05)$.

$*$ significativo a $5 \%$ de probabilidade, $* *$ significativo a $1 \%$ de probabilidade, ns - não-significativo.

$\mathrm{P}=$ cobertura com $5 \mathrm{t} \mathrm{ha}^{-1}$ de palha de cana-de-açúcar; $\mathrm{C}=$ chuva de $30 \mathrm{~mm} ; \mathrm{C}_{12 \mathrm{~h}}=$ chuva de $30 \mathrm{~mm} 12$ horas antes da aplicação; $\mathrm{C}_{2,5}=$ chuva de $2,5 \mathrm{~mm} ; \mathrm{A}=$ aplicação de oxyfluorfen.

Planta Daninha, Viçosa-MG, v. 27, n. 1, p. 197-203, 2009 
Tabela 7 - Controle de Merremia cissoides pelo oxyfluorfen na dose de 3,0 L p.c.ha ${ }^{-1}$, aplicado em diferentes posicionamentos em relação à palha de cana-de-açúcar. BotucatuSP, 2007

\begin{tabular}{|l|c|c|c|c|c|}
\hline \multirow{2}{*}{ Tratamento } & \multicolumn{5}{|c|}{ Dias após a aplicação (DAA) } \\
\cline { 2 - 6 } & 12 & 20 & 26 & 33 & 38 \\
\hline 1. PAC & $97,8 \mathrm{ab}$ & $100,0 \mathrm{a}$ & $100,0 \mathrm{a}$ & $100,0 \mathrm{a}$ & $100,0 \mathrm{a}$ \\
\hline 2. CPA & $99,0 \mathrm{ab}$ & $100,0 \mathrm{a}$ & $100,0 \mathrm{a}$ & $100,0 \mathrm{a}$ & $100,0 \mathrm{a}$ \\
\hline 3. CAP & $99,0 \mathrm{ab}$ & $100,0 \mathrm{a}$ & $100,0 \mathrm{a}$ & $100,0 \mathrm{a}$ & $100,0 \mathrm{a}$ \\
\hline 4. $\mathrm{PC}_{12 \mathrm{~h}} \mathrm{~A}$ & $100,0 \mathrm{a}$ & $100,0 \mathrm{a}$ & $100,0 \mathrm{a}$ & $100,0 \mathrm{a}$ & $100,0 \mathrm{a}$ \\
\hline 5. PAC 2,5 & $96,3 \mathrm{~b}$ & $98,5 \mathrm{~b}$ & $99,5 \mathrm{a}$ & $99,5 \mathrm{a}$ & $99,5 \mathrm{a}$ \\
\hline 6. AC & $99,8 \mathrm{a}$ & $100,0 \mathrm{a}$ & $100,0 \mathrm{a}$ & $100,0 \mathrm{a}$ & $100,0 \mathrm{a}$ \\
\hline 7. Test. palha & - & - & - & - & - \\
\hline 8. Test. s/palha & - & - & - & - & - \\
\hline F tratamento & $5,02 * *$ & $5,40 * *$ & $1,00^{\text {ns }}$ & $1,00^{\text {ns }}$ & $1,00^{\text {ns }}$ \\
CV $(\%)$ & 1,27 & 0,53 & 0,41 & 0,41 & 0,41 \\
\hline DMS & 2,81 & 1,18 & 0,92 & 0,92 & 0,92 \\
\hline
\end{tabular}

Médias seguidas de mesma letra na coluna não diferem estatisticamente entre si pelo teste de Tukey $(\mathrm{P}<0,05)$

$*$ significativo a $5 \%$ de probabilidade, ${ }^{* *}$ significativo a $1 \%$ de probabilidade, ns - não-significativo.

$\mathrm{P}=$ cobertura com $5 \mathrm{t}^{\text {ha }}{ }^{-1}$ de palha de cana-de-açúcar; $\mathrm{C}=$ chuva de $30 \mathrm{~mm} ; \mathrm{C}_{12 \mathrm{~h}}=$ chuva de $30 \mathrm{~mm} 12$ horas antes da aplicação; $\mathrm{C}_{2,5}=$ chuva de $2,5 \mathrm{~mm} ; \mathrm{A}=$ aplicação de oxyfluorfen.

controle desta espécie foi total a partir de 20 DAA. Nesse caso, a quantidade de chuva $(2,5 \mathrm{~mm})$ foi insuficiente para retirada do produto da palha para que pudesse inibir a germinação da planta daninha. Apesar disso, as médias de controle são consideradas excelentes para a condução normal da cultura em campo.

Apesar das variações observadas para as porcentagens de controle nas diferentes datas de avaliação e espécies de plantas daninhas, as diferenças entre os tratamentos se mantiveram ao longo de todo o período de avaliação. De modo geral, o tratamento 3, em que a aplicação do herbicida ocorreu diretamente no solo, recebendo uma camada de palha de $5 \mathrm{t} \mathrm{ha}{ }^{-1}$, o tratamento 4, no qual a aplicação do herbicida ocorreu em palha úmida, e o tratamento 6, com a pulverização ocorrendo sobre o solo sem cobertura de palha após a aplicação, apresentaram os melhores indices de controle das plantas daninhas estudadas.

Pode-se concluir que o herbicida oxyfluorfen demonstrou excelente eficácia no controle de corda-de-viola (Ipomoea grandifolia, Ipomoea quamoclit e Merremia cissoides) e capim-braquiária (Brachiaria decumbens), independentemente do posicionamento na palha ou no solo, demonstrando assim ser uma excelente opção para o controle de plantas daninhas em cana-de-açúcar em sistema de cana-crua ou queimada.

\section{AGRADECIMENTOS}

À FAPESP, pelo suporte técnico e financeiro para execução deste trabalho.

\section{LITERATURA CITADA}

AZANIA, A. A. P. M. et al. Interferência de palha de canade-açúcar (Saccharum spp.) na emergência das espécies de plantas daninhas da família Convolvulaceae. Planta Daninha, v. 20, n. 1, p. 207-212, 2002

CORREA, M. R. Dinâmica e eficácia da mistura formulada de diuron e hexazinone no sistema de produção de cana crua. 2006. 150 f. Tese (Doutorado em Agricultura/Produção Vegetal) - Universidade Estadual Paulista, Botucatu, 2006.

CORREIA, N. M.; DURIGAN, J. C. Emergência de plantas daninhas em solo coberto com palha de cana-de-açúcar. Planta Daninha, v. 22, n. 1, p. 11-17, 2004

JONES JR., R. E. et al. Alachlor and metribuzin movement and dissipation in a soil profile as influenced by soil surface condition. Weed Sci., v. 38, n. 6, p. 589-97, 1990

LAMOREAUX, R. J. et al. Efficacy of dimethenamid, metolachlor and encapsulated alachlor in soil covered with crop residue. Weed Sci., v. 3, p. 1015-20, 1993

LOCKE, M. A.; BRYSON, C. T. Herbicide-soil interaction in reduced tillage and plant residue management systems. Weed Sci., v. 45, n. 2, p. 307-20, 1997.

MARTINS, D. et al. Emergência em campo de dicotiledôneas infestantes em solo coberto com palha de cana-de-açúcar. Planta Daninha, v. 17, n. 1, p. 151-161, 1999.

MEDEIROS, D.; CHRISTOFFOLETI, P. J. Efeito da intensidade de chuva e da quantidade de palha de cana-deaçúcar sobre a eficácia de herbicidas. In: CONGRESSO BRASILEIRO DA CIÊNCIA DAS PLANTAS DANINHAS, 23., 2002, Gramado. Anais... Gramado: Sociedade Brasileira de Ciência do Solo, 2002. p. 510.

NEGRISOLI, E. Associação do herbicida tebuthiuron com a cobertura de palha no controle de plantas daninhas no sistema de cana-crua. 2004. 88 f. Tese (Doutorado em Agronomia - Proteção de Plantas) - Universidade Estadual Paulista, Botucatu, 2005. 
NEGRISOLI, E. et al. Controle de plantas daninhas pelo amicarbazone aplicado na presença de palha de cana-deaçúcar. Planta Daninha, v. 25, n. 3, p. 603-611, 2007 a.

NEGRISOLI, E. et al. Associação do herbicida tebuthiuron com a cobertura de palha no controle de plantas daninhas no sistema de cana-crua. Planta Daninha, v. 25, n. 3 , p. $621-628,2007 \mathrm{~b}$.

NEGRISOLI, E. et al. Eficácia do sulfentrazone em diferentes doses associado à palha de cana-de-açúcar com ou sem chuva após a aplicação no controle de plantas daninhas. In: CONGRESSO BRASILEIRO DA CIÊNCIA DAS PLANTAS DANINHAS, 24., 2004, São Pedro. Anais... São Pedro: Sociedade Brasileira da Ciência do Solo, 2004. p. 150.

ROSSI, C. V. S. Eficácia no controle de plantas daninhas pelos herbicidas metribuzin e isoxaflutole aplicados isolados ou em mistura na cultura da cana-de-açúcar (cana crua) 2007. 87 f. Tese (Doutorado em Agricultura/ Produção Vegetal) - Universidade Estadual Paulista, Botucatu, 2007.
SOCIEDADE BRASILEIRA DA CIENNCIA DAS PLANTAS DANINHAS - SBCPD. Procedimentos para instalação, avaliação e análise de experimentos com herbicidas. Londrina: 1995.42 p.

SORENSON, B. A. et al. Effects of tillage, application time and rate on metribuzin dissipation. Weed Res., v. 31, n. 6, p. 333-345, 1991.

TOFOLI, G. R. Deposição e lixiviação do herbicida tebuthiuron em palha de cana-de-açúcar. 2004. 63 f. Tese (Doutorado em Proteção de Plantas/Produção Vegetal) Universidade Estadual Paulista, Botucatu, 2004.

VEIGA FILHO, A. Evolução da mecanização do corte da cana-de-açúcar em São Paulo. In: CONGRESSO NACIONAL DA STAB, 8., 2002, Recife. Anais... Recife: Sociedade Brasileira da Ciência das Plantas Daninhas, 2002. p. 515-521.

VELINI, E. D.; NEGRISOLI, E. Controle de plantas daninhas em cana crua. In: CONGRESSO BRASILEIRO DA CIÊNCIA DAS PLANTAS DANINHAS, 22., 2000, Foz de Iguaçu. Palestras... Foz de Iguaçu: Sociedade Brasileira da Ciência das Plantas Daninhas, 2000. p. 148-164. 